\title{
A recipe for practical full waveform inversion in Orthorhombic anisotropy
}

Tariq Alkhalifah, Nabil Masmoudi, and Ju-won Oh, KAUST

\section{SUMMARY}

Multi parameter full waveform inversion (FWI) usually suffers from the inherent tradeoff in the multi parameter nature of the model space. In orthorhombic anisotropy, such tradeoff is magnified by the large number of parameters involved in representing the elastic or even the acoustic approximation of such a medium. However, using a new parameterization with distinctive scattering features, we can condition FWI to invert for the parameters the data are sensitive to at different stages, scales, and locations in the model. Specifically, with a combination made up of a velocity and particular dimensionless ratios of the elastic coefficients, the scattering potential of the anisotropic parameters have stationary scattering radiation patterns as a function of the type of anisotropy. With our new parametrization, the data is mainly sensitive to the scattering potential of 4 parameters: the horizontal velocity in the $x_{1}$ direction, $\epsilon$, which provides scattering mainly near zero offset in the $x_{1}-x_{3}$ vertical plane, $\epsilon_{d}$, which is the ratio of the horizontal velocity squared in the $x_{1}$ and $x_{2}$ direction, and $\delta_{3}$ describing the anellipticity in the horizontal plane. Since, with this parametrization, the radiation pattern for the horizontal velocity and $\epsilon$ is azimuth independent, we can perform an initial VTI inversion for these two parameters, and then use the other two parameters to fit the azimuth variation in the data. This can be done at the reservoir level or any region of the model. Including the transmission from reflections, the migration velocity analysis (MVA) component, into the picture, the multi azimuth surface seismic data are mainly sensitive to the long wavelength components of $v_{h}, \delta_{3}$, and $\epsilon_{d}$ through the diving waves, and $\eta_{1}, \eta_{d}$, and $\delta_{3}$, in the transmission to or from reflectors (especially, in the presence of large offsets). They are also sensitive to the short wavelength component of $v_{h}$ and $\epsilon$.

\section{INTRODUCTION}

Orthorhombic anisotropy has emerged as the most practical model that combines the natural, mostly vertical, layering of the Earth (due to gravity), and the normal horizontal stresses from salt intrusions, tectonic regimes, as well as reservoir level fracturing, and yet the model is reasonably treatable (Cheadle et al., 1991; Schoenberg and Helbig, 1997). We may not invert for all the parameters, but using waveform inversion we may invert for the parameters we need and the parameters that the data are sensitive to. In 3D multi azimuth acquisition, we have sensitivities along the vertical direction usually provided by the reflectivity, sensitivities in the polar direction usually highlighted by the data varia- tion with offset, sensitivities to lateral changes given by the position of the analysis, and finally sensitivities to the azimuth provided directly by the azimuth of the acquisition. Two of these sensitivities are mainly related to inhomogeneity, and the other two are related to anisotropy. Orthorhombic anisotropic inhomogeneous media can encompass all these variations. Since, $P$ wave data are the easiest to acquire and have the highest quality, the acoustic assumption can reduce the complexity of the inversion considerably (Alkhalifah, 2003; Song and Alkhalifah, 2013). However, in FWI, the elastic story is useful if we are heading toward higher resolution (Tarantola, 1986), where amplitudes matter. Even in the acoustic case, six parameters that may change laterally or vertically govern the orthorhombic model description (Alkhalifah, 2003). Even with multi azimuth data, the problem is complicated with complex tradeoff in the data sensitivity to these parameters. In addition, if the azimuth of the horizontal symmetry axis is unknown, we will need to invert for it as well. As a result, we have a potential for a large Null space in the inverse problem.

However, we have realized recently that these parameters can be inverted at different scales, within the surface $\mathrm{P}$-wave seismic data wavelength range (Masmoudi and Alkhalifah, 2016). We tend to have a reflectivity and a traveltime resolution scale. The data are sensitive to these parameters (other than velocity) at mainly one resolution scale, and thus, that is what we can constrain. In addition, the sensitivity of the data to perturbations in the model parameters is well represented by the first (linear) term of the Born series, which adjointly describes the gradients used in FWI. However, data are also very sensitive to the transmission components of these model parameters to and from predicted perturbations, and are well approximated by the long wavelength part of the second term of the Born series. This nonlinear component of the influence is usually handled under the context of migration velocity analysis (MVA) in the image domain (Yilmaz and Chambers, 1984), or recently by reflection waveform inversion (RWI) in the data domain (Xu et al., 2012). In this abstract, we will focus on analyzing the scattering and transmission potential behavior of the model parameters using a new parametrization for orthorhombic anisotropy. In this case, we analyze inversion gradients corresponding to classical scattering and those corresponding to transmission from a predicted reflector associated with RWI or MVA.

\section{A NEW PARAMETRIZATION}

Masmoudi and Alkhalifah (2016) analyzed the radiation patterns of the scattering potential for many parameter- 


\section{Orthorhombic anisotropy optimal parametrization}

izations for an orthorhombic assumption of the medium and ended up introducing a new parametrization based on deviation parameters with potentially attractive features for FWI and MVA. We will start by reviewing this new parametrization and their corresponding radiation patterns. We will look at their transmission and scattering features.

Guided by the insights presented in Alkhalifah and Plessix (2014), we concluded that a parameterization given by one velocity (or two in the elastic case), and dimensionless parameters allows for continuity of the scattering potential of key parameters as we move from higher symmetry anisotropy to lower ones. For the acoustic case, the central parameter in the suggested parametrization is the horizontal, $v_{h}=v_{h 1}$ (or for RWI, the NMO, $v_{n}=v_{n 1}$ ) velocity in the $x_{1}-x_{3}$ (or $x-z$ ) plane, where $v_{h}^{2}=v_{v}^{2}(1+2 \epsilon)$, and $v_{n}^{2}=v_{v}^{2}(1+2 \delta)$, with $\delta=\delta_{1}$ and $\epsilon=\epsilon_{1}$ defined in the same plane. Here, $v_{v}$ is the vertical $P$-wave velocity. The subscript number convention used here differ from Tsvankin (1997), who used the normal to the plane to define the parameters, and inline with Alkhalifah (2003). Thus, we remove the subscript to mitigate the confusion. Alkhalifah (2016) suggested that the $v_{h}, \eta$, and $\epsilon$ combination were optimal for FWI, and $v_{n}, \eta$, and $\delta$ combination were optimal for MVA in acoustic VTI media. In both of these parameterizations that include a central velocity and two dimensionless parameters, the scattering features of the central velocity is the same as that for isotropic materials (not changing with polar angle), which allowed for a continuity between an isotropic assumption of the medium and a VTI one. Accordingly, with similar parametrization, we can utilize orthorhombic parameters that maintain the VTI scattering features regardless of azimuth.

In the acoustic orthorhombic case, we will need an additional three parameters. Thus, defining the parameters with respect to one of the VTI planes (deviation parameters) allows the scattering from the VTI parameters to be azimuth independent (Masmoudi and Alkhalifah, 2016). Along with $\delta_{3}$, which is the $\delta$ parameter defined from the $x$-axis along the TI horizontal plane, we can utilize two of

$$
\begin{aligned}
\delta_{d} & =\frac{1}{2}\left(\frac{v_{n 2}^{2}}{v_{n 1}^{2}}-1\right), \\
\epsilon_{d} & =\frac{1}{2}\left(\frac{v_{h 2}^{2}}{v_{h 1}^{2}}-1\right) . \\
\eta_{d} & =\frac{\epsilon_{d}-\delta_{d}}{1+2 \delta_{d}},
\end{aligned}
$$

where $v_{h 2}$ and $v_{n 2}$ are the horizontal and NMO velocities in the $x_{2}-x_{3}(y-z)$ plane. This is the last time you will hear about these velocities in this abstract. We centralize the acoustic orthorhombic description with one velocity. The choice of the two deviation parameters depend on this central velocity. For the $v_{h}$ combination, we use $\eta_{d}$ and $\epsilon_{d}$, and for the $v_{n}$ combination, we use $\delta_{d}$ and $\epsilon_{d}$ for reasons given in Masmoudi and Alkhalifah (2016)) and described below. The parameters $\epsilon_{d}$, and $\eta_{d}$, as well as $\delta_{3}$ are dimensionless as they describe the deviation from VTI and if we set them to zero, the medium reduces to VTI. Using this parametrization, Masmoudi and Alkhalifah (2016) derived radiation patterns for acoustic orthorhombic media. We analyze those radiation patterns next and extract some interesting insights for inversion.

\section{THE SCATTERING POTENTIAL OF THE PA- RAMETERS (FWI)}

We focus on the scattering potential from a horizontal reflector. This scattering potential is defined as a function of the scattering angle, $\theta$, the angle between the background (source) and the scattered (receiver) wave paths measured at the scattering point. A scattering angle of zero implies mainly zero offset reflection data, while $\theta=180^{\circ}$ implies direct or diving waves. For our $3 \mathrm{D}$ orthorhombic case, the scattering potential (radiation pattern) is also dependent on the azimuth of the incident and scattered wave paths at the scattering point. The azimuth is measured from the $x_{1}$ (or $x$ ) axis of the orthorhombic anisotropy. The resolvability of the desired scattering object is furnished by diffraction tomography principles in which the model wavenumber vector magnitude is proportional to the frequency, $\omega$, but also proportional to the cosine of half of the scattering angle (Miller et al., 1987; Jin et al., 1992; Thierry et al., 1999), given: $\left|\mathbf{k}_{\mathbf{m}}\right|=2 \frac{\omega}{v_{0}} \cos \frac{\theta}{2}$, where $v_{0}$ is the velocity at the scattering point of the background isotropic model. As a result, we expect large reflection scattering angle to produce lower resolution. Keeping this relation in mind we analyze the radiation patterns shown in Figures 1a$1 d$.

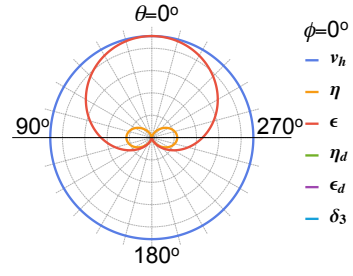

(a)

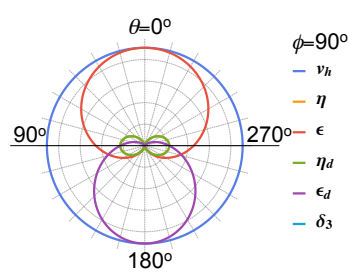

(c)

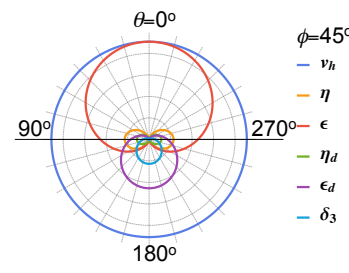

(b)

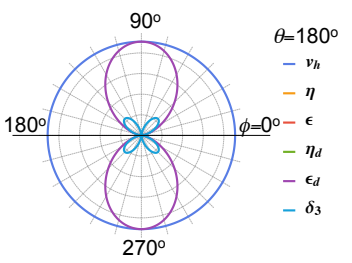

(d)
Figure 1: Radiation patterns of the acoustic orthorhombic model parameters as a function of scattering angle, $\theta$, for various azimuths $\phi(\mathrm{a}-\mathrm{c})$, and $\mathrm{d})$ the radiation pattern for a 180 degree scattering angle as a function of azimuth (like a horizontal slice of a-c). 


\section{Orthorhombic anisotropy optimal parametrization}

As mentioned, the scattering potential of the central velocity parameter is isotropic over scattering angles and azimuths (Figure 1d). Both $\eta$ and $\epsilon$ though produce scattering at certain polar angles, the scattering is stationary with azimuth. As suggested by Alkhalifah (2016), for surface seismic data $\eta$ has low scattering energy focussed mainly at the far offsets. It probably can be ignored at the acoustic stage. Meanwhile, $\epsilon$ admits scattering at small offsets, and can be used to match the amplitudes to overcome the limitations of the acoustic assumption. As we leave zero azimuth (Figures $1 \mathrm{~b}$ and $1 \mathrm{c})$, the orthorhombic parameters, $\epsilon_{d}, \eta_{d}$, and $\delta_{3}$ start to induce scattering. While the $\delta_{3}$ influence resides in the middle azimuths (around $\phi=45^{\circ}$ ), the other two parameters have their maximum influence at $\phi=90^{\circ}$. Again, the $\eta_{d}$ influence resides mainly at large offsets, and might be ignored at the acoustic stage. Meanwhile, $\epsilon_{d}$ addresses the variation in the horizontal velocity mainly for diving waves in the $\phi=90^{\circ}$ direction. In the elastic case, where amplitudes matter, and with reasonable offsets in the acquired data, $\eta$ and $\eta_{d}$ could play a role in describing the scattering variation away from vertical.

Since the maximum perturbation in the deviation parameters resides at $\phi=90^{\circ}$, we can use this fact to search for the azimuth of the vertical orthorhombic anisotropy. With wide or full azimuth data, we scan over orthogonal azimuths to find the pair that admit the largest $\epsilon_{d}$ gradient energy. Theoretically, this azimuth combination represent the symmetry axes of the orthorhombic anisotropy. However, since $\epsilon_{d}$ induce scattering at larger angles (Figure 1c), the expected resolution of the inverted azimuth direction is low.

\section{THE TRANSMISSION COMPONENT FROM A REFLECTION (MVA)}

The long wavelength component of the velocity model, or the anisotropic parameters, typically control the majority of wave propagation characteristics, effecting the shape of the waves, which is conveniently described, in the high frequency asymptotic, by traveltimes. To analyze the behavior of this component for reflections, we focus on the transmission $\left(\theta=180^{\circ}\right)$ to a horizontal reflector. However, the depth of this reflector also depends on the background velocity. Since the background medium is assumed isotropic, we scale the vertical axis by the background velocity, which is assumed to equal the vertical velocity since we are dealing with horizontal reflectors (Alkhalifah et al., 2001; Plessix, 2013).

Thus, we mitigate the effect of the depth of the reflector by mapping the vertical axis, $z$, to vertical time using the relation $d z=v_{v} d \tau$. The resulting wave equation for orthorhombic anisotropy becomes vertical velocity (and $\delta$ ) independent. As expected, the transmission from the Earth surface in the vertical direction to a horizontal reflector (zero offset) does not include long wavelength information. Only, multi offset data, as is

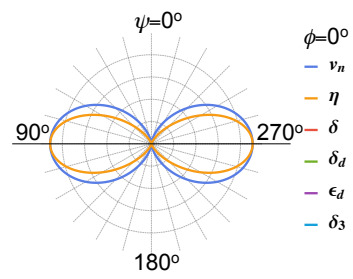

(a)

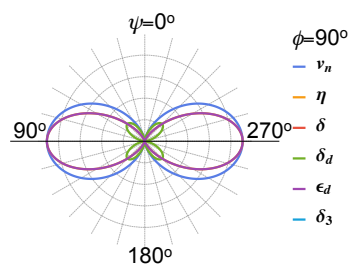

(c)

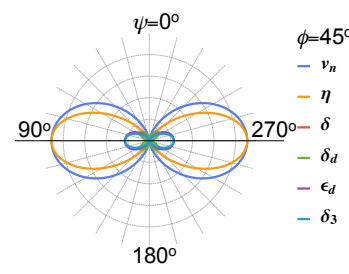

(b)

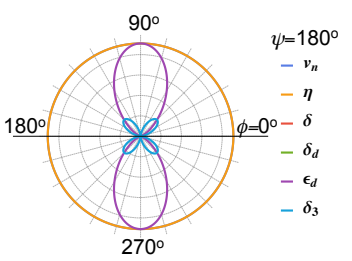

(d)
Figure 2: Radiation patterns of the acoustic orthorhombic model parameters as a function of transmission angle, $\psi$, for various azimuths $\phi(\mathrm{a}-\mathrm{c})$, and $\mathrm{d})$ the radiation pattern for a 180 degree transmission angle as a function of azimuth (like a horizontal slice of a-c). Same as Figure 1d, but with different paramerization

generally known, allow for resolution of velocity, and in orthorhombic anisotropic media that velocity is $v_{n}$ in the $x$ direction, with $\delta_{d}$ describing the NMO velocity deviation for the $y$ direction. Larger offsets should illuminate long wavelength $\eta$ in the $x$ direction, and $\epsilon_{d}$, which represents the deviation in the nonhyperbolic moveout in the $y$ direction. While $\delta_{3}$ requires large offsets in the intermediate azimuths. These intuitive statements are reflected in the transmission radiation patterns for the $v_{n}$ parameter combination corresponding to scaling the vertical axis to vertical time shown in Figures 2a-2c. Since these radiation patterns are extracted using the Born linearized data sensitivity to perturbations, these particular ones highlight the MVA, including RWI, resolvability of the orthorhombic parameters. As expected, when transmission occurs vertically (from horizontal reflectors), the data are insensitive to all the parameters. This is courtesy of the velocity-depth ambiguity in that direction. In fact, part of the requirement for a successful RWI free of reflectivity imprint is that we match the data perfectly at zero offset in the demigration. Thus, the residual for the zero offset path is zero, and the resulting gradient for that path has generally zero energy. In the zero-azimuth $(x)$ direction (Figure $2 \mathrm{a}$ ), the influence of $v_{n}$ appears at moderate transmission angles $\psi$, while $\eta$ requires larger angles. Using the deviation parameters to represent the orthorhombic model, $v_{n}$ and $\eta$ scattering potentials are stationary with azimuth (Figures $2 \mathrm{~b}$ and $2 \mathrm{c}$ ). Actually, for 180 degree transmission path (horizontal wave path), the two coincide as demonstrated in Figure 2d. Thus, if we manage to resolve $v_{n}$ and $\eta$ using any azimuth (they are fully accurate when 


\section{Orthorhombic anisotropy optimal parametrization}

that azimuth is 0 ), we can invert for the long wavelength transmission $\delta_{d}$ (moderate transmission angles) and $\epsilon_{d}$ (large transmission angles) using an orthogonal azimuth. As previously mentioned, these two orthogonal azimuths should coincide with zero and 90 degree azimuths of the orthorhombic anisotropy when $\delta_{d}$ and $\epsilon_{d}$ have there maximum potential value for the model. The intermediate azimuth large offset data may illuminate long wavelength $\delta_{3}$ (Figure-2d).

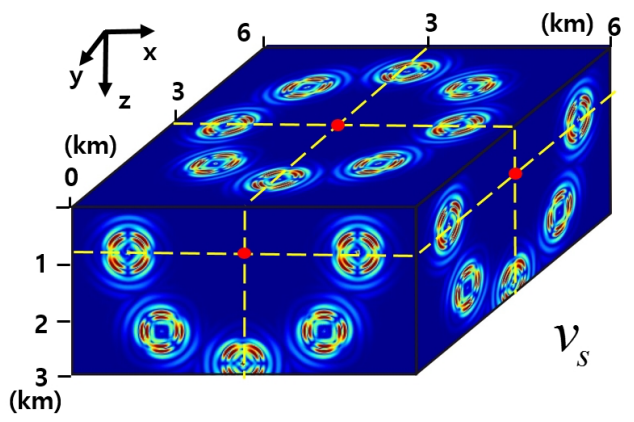

(a)

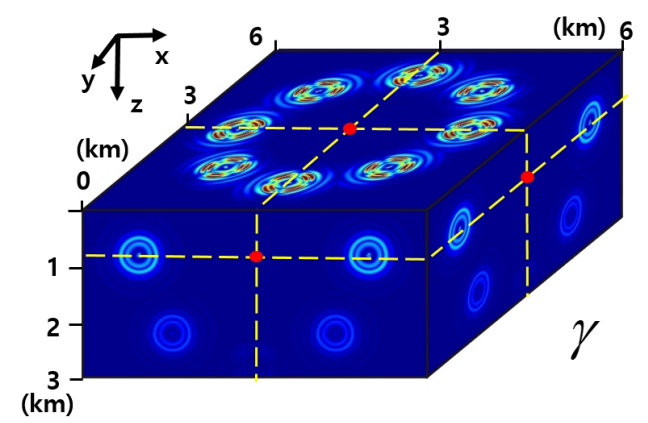

(b)

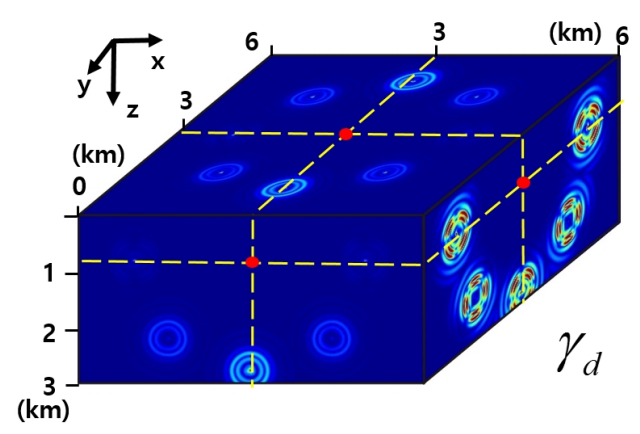

(c)

Figure 3: The wavefield response to thirteen scatterers placed at equal distances from the source (Red dot). The scatterers correspond to a) $v_{s}$, b) $\gamma$, and c) $\gamma_{d}$ perturbations.

\section{THE ELASTIC CASE}

The elasticity of the Earth matters for P-waves in the above analysis for the scattering case, as transmissions for acoustic and elastic P-waves are similar. So the elastic nature of the Earth has minor influence on the MVA part of our inversion for long wavelength model information. The true effect of ignoring the elastic nature of the Earth appears mainly in the inversion for scattering (FWI). Considering an elastic orthorhombic assumption of the medium, we will need to add three parameters to the description of the model (Oh and Alkhalifah, 2016), over what we used in the acoustic case. These elastic parameters are the shear wave velocity $v_{s}$ in vertical direction for a polarization in the $x$ direction, $\gamma=\gamma_{1}$, and a deviation parameter $\gamma_{d}$ given by

$$
\gamma_{d}=\frac{\gamma_{1}-\gamma_{2}}{1+2 \gamma_{2}}
$$

Here, $C_{66}=\left(1+2 \gamma_{1}\right) C_{55}=\rho\left(1+2 \gamma_{1}\right) v_{s}^{2}$, and $C_{66}=$ $\left(1+2 \gamma_{2}\right) C_{44}$, where $C_{44}, C_{55}$, and $C_{66}$ are the elastic coefficients corresponding to shear waves (Tsvankin, 1997). An interesting feature of representing the elastic parameters by $v_{s}, \gamma$, and $\gamma_{d}$, we maintain the radiation patterns we ended up with for $P$-waves in the acoustic case.

The wavefield response to perturbations in the elastic parameters, $v_{s}, \gamma$, and $\gamma_{d}$, can be analyzed by visualizing snap shots of the scattered wavefield from scatterers strategically distributed radially and along all azimuths as shown in Figures 3a-3c. The scatterers are placed at equal distances from a source in the middle (red dot). The main "take away" from these Figures is the angles in which scattering occurs, and specifically the variation between $P$-waves (faster) and $S$ waves (slower), as they are related to diffraction patterns. For $v_{s}$ perturbations, there are a clear symmetry radially and along all azimuths for both $P$ - and $S$ - waves, with respect to the source location. For $\gamma$ perturbations, we have mainly $S$ wave scattering, as evident by the wavefield size and the energy of the scattering reduces in the vertical direction. Finally, the $\gamma_{d}$ scatterers reflect the azimuthal preference as scattering energy dissipates in the $x$-direction, especially for $P$-waves.

\section{CONCLUSIONS}

Through the analysis of the data dependency on the scattering and transmission components of a particular (we think optimal) anisotropic parameter representation of Orthorhombic anisotropy, we managed to understand our inversion limitations, and outline a strategy for a practical inversion of Orthorhombic anisotropy using multi-azimuth surface seismic data acquisition. The combinations we used here given by either $v_{h}$ or $v_{n}$ and the corresponding dimensionless parameters served to allow for minimum tradeoff for FWI and MVA methods, respectively. Handling the azimuth variation as a deviation from a VTI plane allowed the VTI parameters to have stationary scattering potential with azimuth, and thus, decouple the azimuth dependency to the new deviation parameters. A similar approach is utilized in the elastic stage allowing for numerous strategies to address the mutli-parameter nature of the model inversion. 
EDITED REFERENCES

Note: This reference list is a copyedited version of the reference list submitted by the author. Reference lists for the 2016 SEG Technical Program Expanded Abstracts have been copyedited so that references provided with the online metadata for each paper will achieve a high degree of linking to cited sources that appear on the Web.

\section{REFERENCES}

Alkhalifah, T., 2003, An acoustic wave equation for orthorhombic anisotropy: Geophysics, 68, 11691172, http://dx.doi.org/10.1190/1.1598109.

Alkhalifah, T., 2016, Research note: Insights into the data dependency on anisotropy: An inversion prospective: Geophysical Prospecting, 64, 505-513, http://dx.doi.org/10.1111/1365-2478.12345.

Alkhalifah, T., S. Fomel, and B. Biondi, 2001, The space-time domain: theory and modelling for anisotropic media: Geophysical Journal International, 144, 105-113, http://dx.doi.org/10.1046/j.1365-246x.2001.00300.x.

Alkhalifah, T., and R. E. Plessix, 2014, A recipe for practical full-waveform inversion in anisotropic media: An analytical parameter resolution study: Geophysics, 79, no. 3, R91-R101, http://dx.doi.org/10.1190/geo2013-0366.1.

Cheadle, S. P., R. J. Brown, and D. C. Lawton, 1991, Orthorhombic anisotropy: A physical seismic modeling study: Geophysics, 56, 1603-1613, http://dx.doi.org/10.1190/1.1442971.

Jin, S., R. Madariaga, J. Virieux, and G. Lambaré, 1992, Two-dimensional asymptotic iterative elastic inver-sion: Geophysical Journal International, 108, 575-588, http://dx.doi.org/10.1111/j.1365246X.1992.tb04637.x.

Masmoudi, N., and T. Alkhalifah, 2016, Scattering potential of acoustic orthorhombic parametrization An inversion prospective: 78th Annual International Conference and Exhibition, EAGE, Extended Abstracts, http://dx.doi.org/10.3997/2214-4609.201601010.

Miller, D., M. Oristaglio, and G. Beylkin, 1987, A new slant on seismic imaging: Migration and integral geometry: Geophysics, 52, 943-964, http://dx.doi.org/10.1190/1.1442364.

Oh, J., and T. Alkhalifah, 2016, A hierarchical parameterisation for elastic orthorhombic anisotropic parameter inversion: 78th Annual International Conference and Exhibition, EAGE, Extended Abstracts, http://dx.doi.org/10.3997/2214-4609.201601006.

Plessix, R., 2013, A pseudo-time formulation for acoustic full waveform inversion: Geophysical Journal International, 192, no. 2, 613-630, http://dx.doi.org/10.1093/gji/ggs056.

Schoenberg, M., and K. Helbig, 1997, Orthorhombic media: Modeling elastic wave behavior in a vertically fractured earth: Geophysics, 62, no. 6, 1954-1974, http://dx.doi.org/10.1190/1.1444297.

Song, X., and T. Alkhalifah, 2013, Modeling of pseudoacoustic P-waves in orthorhombic media with a low-rank approximation: Geophysics, 78, no. 4, C33-C40, http://dx.doi.org/10.1190/geo20120144.1.

Tarantola, A., 1986, A strategy for nonlinear elastic in-version of seismic reflection data: Geophysics, 51, 1893-1903, http://dx.doi.org/10.1190/1.1442046.

Thierry, P., S. Operto, and G. Lambare, 1999, Fast 2-D ray born migration/inversion in complex media: Geophysics, 64, 162-181, http://dx.doi.org/10.1190/1.1444513.

Tsvankin, I., 1997, Anisotropic parameters and p-wave velocity for orthorhombic media: Geophysics, 62, 1292-1309, http://dx.doi.org/10.1190/1.1444231.

Xu, S., D. Wang, F. Chen, G. Lambare, and Y. Zhang, 2012, Inversion on reflected seismic wave: 82nd Annual International Meeting, SEG, Expanded Abstracts, 1-7.

Yilmaz, O., and R. Chambers, 1984, Migration velocity analysis by wavefield extrapolation: Geophysics, 49, 1664-1674, http://dx.doi.org/10.1190/1.1441574. 